Interventions (or Assessment of Risk Factors) Risk of reinjury and osteoarthritis following knee surgery in professional folk dancers.

Results The dancers suffered 14 knee injuries requiring arthroscopic surgery (3 meniscus tears, 4 anterior cruciate ligament tears one of which is with posterolateral corner tear, 1 posterior cruciate ligament tear, 1 patellar dislocation, 1 infrapatellar bursitis, 2 Hoffa's fat pad syndromes, 2 symptomatic medial plicaes) during a ten-year period.Following surgeries, the dancers could restart to perform live on the stage in 19,5 \pm 12 (range:5 to52) weeks on average. Injuries and postoperative times to return to dance was $56,7 \pm 23$ (26-108) months to follow-up with the same clinic and same surgeon for the patients. One dancer had reoperation due to meniscus retear after 4 years. The rate of reinjury is $7.14 \%$ after knee surgery. All of the dancers who underwent arthroscopic knee surgery were evaluated for osteoarthritis according to the Kellgren Lawrence classification. The osteoarthritis were classified as G:0 in 7 patients, G:1 in 3 patients, and G:2 in 4 patients on final knee radiographs. All of the operated patients continued with their careers in dancing.

Conclusions Knee surgeries for the cases mentioned above do not necessarily put an end to their dancing career. This may be attributed to the dancers' balance and coordination skill focus compared to endurance focus during training.

\section{BONE MINERAL DENSITY AND ASSOCIATED FACTORS: DO YOUNG FEMALE DANCERS AND OTHER RECREATIONAL SPORT ATHLETES DIFFER?}

\begin{abstract}
${ }^{1}$ Meghan Critchley, ${ }^{2}$ Clodagh Toomey, ${ }^{3}$ Stacey M Lobos, ${ }^{1,2,4}$ Luz Palacios-Derflingher, 1,2,5Sarah J Kenny, ${ }^{1,2,3,4,5}$ Carolyn Emery. 'Sport Injury Prevention Research Centre, Faculty of Kinesiology, University of Calgary, Calgary, Alberta, Canada; ${ }^{2}$ Alberta Children's Hospital Research Institute, University of, Calgary, Alberta, Canada; ${ }^{3}$ Human Performance Laboratory, Faculty of Kinesiology, Calgary, Alberta, Canada; ${ }^{4}$ Department of Community Health Sciences, Cumming School of Medicine, Calgary, Alberta, Canada; ${ }^{5} O^{\prime}$ Brien Institute for Public Health, University of Calgary, Calgary, Alberta, Canada
\end{abstract}

\subsection{6/bjsports-2021-IOC.116}

Background As an aesthetic activity, dancers are susceptible to Relative Energy Deficiency in Sport (RED-S), specifically low bone mineral density (BMD). Compared to sedentary groups, dancers have significantly higher BMD, but little is known about how BMD in dancers relates to other athletic populations. This knowledge may better inform comparative values and risk of bone injury.

Objective To evaluate associated factors of BMD among female dancers and sporting athletes.

Design Cross-sectional study.

Setting Human Performance Lab, University of Calgary.

Patients (or Participants) 275 females [138 pre-professional dancers (18.4 years IQR: 15.3, 19.9); 137 recreational sport athletes (23.2 years IQR: 21.9, 24.7)] participated.

Interventions (or Assessment of Risk Factors) Factors evaluated: Age (years), BMI $\left(\mathrm{kg} / \mathrm{m}^{2}\right)$, supplement intake (Ca+, vitD; yes/ no), stress fracture history (yes/no), irregular menses (yes/no), and MRI/bone scan (yes/no), one-year injury history (yes/no), and activity (dance/sport). All two-way interactions were evaluated.

Main Outcome Measurements Whole-body BMD $\left(\mathrm{g} / \mathrm{cm}^{2}\right)$ was estimated from dual-energy x-ray absorptiometry. Lower extremity (LE) segmentation followed published protocols.
Results The mean whole-body BMD was $1.03 \mathrm{~g} / \mathrm{cm}^{2}$ (SD:0.10) in dancers and $1.14 \mathrm{~g} / \mathrm{cm} 2$ (SD:0.08) in athletes. LE BMD was $1.10 \mathrm{~g} / \mathrm{cm}^{2} \quad$ (SD:0.11) in dancers and $1.19 \mathrm{~g} / \mathrm{cm} 2$ (SD:0.08) in athletes. Age was positively associated with whole-body BMD $(b=0.008,97.5 \% \mathrm{CI} ; 0.004,0.012)$ and LE BMD $(b=0.007,97.5 \% C I ; 0.002,0.010)$. Activity modified the relationship between $\mathrm{BMI}$ and BMD $(\mathrm{p}<0.001)$, for example, for a BMI of $20.1 \mathrm{~kg} / \mathrm{m}^{2}$ (mean of participants) the difference between dancers and athletes is $-0.389 \mathrm{~g} / \mathrm{cm}^{2}[97.5 \% \mathrm{CI}$ : $0.566,-0.195]$ and $-0.381 \mathrm{~g} / \mathrm{cm}^{2}$ [97.5\% CI: $\left.-0.552,-0.197\right]$ for whole body and LE BMD respectively. No other variables were associated with BMD.

Conclusions Older age is associated with higher BMD in female pre-professional dancers and sport athletes. The relationship between BMI and BMD depends on activity type for both whole-body and lower extremity sites. Efforts to prevent bone injury should focus on younger dancers and athletes and consider the associations of changing body composition over a training season with low BMD.

\section{INJURY ASSOCIATED WITH DANCE EDUCATION: A SYSTEMATIC REVIEW}

${ }^{1}$ Meghan Critchley, ${ }^{1,2,3}$ Sarah Kenny, ${ }^{4}$ Ashleigh Ritchie, ${ }^{4,5}$ Katy Chambers, ${ }^{6}$ Carly D McKay. ${ }^{1}$ Sport Injury Prevention Research Centre, Faculty of Kinesiology, University of Calgary, Calgary, Alberta, Canada; ${ }^{2}$ Alberta Children's Hospital Research Institute, University of Calgary, Calgary, Alberta, Canada; ${ }^{3} \mathrm{O}^{\prime}$ Brien Institute for Public Health, University of Calgary, Calgary, Alberta, Canada; ${ }^{4}$ Royal Academy of Dance, London, UK; ${ }^{5}$ Trinity Laban Conservatoire of Music and Dance, London, UK; ${ }^{6}$ Department for Health, University of Bath, Claverton Down, Bath, UK

\subsection{6/bjsports-2021-IOC.117}

Background Several studies have investigated injury in specific genres (i.e., ballet, contemporary) and/or levels (i.e., pre-professional, professional) of dance. Less is known about injuries sustained during the teaching of dance, which accounts for the highest proportion of the global dance community.

Objective To determine the risk of injury associated with participation in organized dance education.

Design Systematic review.

Setting Formal dance education (e.g., community classes, dance schools/studios, university programs).

Patients (or Participants) Dance students and teachers.

Main Outcome Measurements Six electronic databases were searched to August 2020 (Medline, EMBASE, SportDiscus, CINAHL, SCOPUS, Cochrane). Inclusion criteria were: original data from dance teacher/student samples, injury and exposure related to formal dance classes, any dance genre. Studies were excluded if no estimate of exposure was reported, if injuries occurred during rehearsal/performance, or if dance was used as a therapeutic intervention or for exercise. Two reviewers independently assessed each paper for inclusion at abstract and full text screening stages. Study quality was assessed using the Joanna Briggs Institute Level of Evidence tool.

Results Twenty-five papers were included.

Most studies $(n=22)$ focused on dance students only, two included only dance teachers, and one study included both. The quality of studies ranged from poor to moderate. For students and teachers, most injuries were overuse/chronic and involved the lower limb. Student injury rates were estimated at $0.8-9.3$ injuries $/ 1000 \mathrm{~h}$. In dance teachers, a single study described 732 pain reports/1000 $\mathrm{h}$ and injury incidence 
estimates ranged from $0.86-1.25$ injuries/dance teacher. Due to limited investigation of risk factors, results could not be stratified by sex, age, or dance style.

Conclusions There have been few primary investigations of injury in dance education settings, despite large rates of dance participation. Consistent injury and exposure definitions, and high-quality prospective studies are needed for examining injury risk in the dance teaching environment.

\section{ASSOCIATION BETWEEN BASELINE FACTORS AND RISK OF INJURY AMONGST PRE-PROFESSIONAL DANCERS}

1,2,35arah J Kenny, ${ }^{1} \mathrm{KV}$ Vineetha Warriya, ${ }^{1,4}$ Luz Palacios-Derflingher, ${ }^{5}$ Jackie Whittaker, 1,2,6 Carolyn Emery, 'Meghan Critchley. 'Sport Injury Prevention Centre, Faculty of KinesiologyUniversity of Calgary, Calgary, Canada; ${ }^{2}$ Alberta Children's Hospital Research Institute, University of Calgary, Calgary, Canada; ${ }^{3} O^{\prime} B r i e n$ Institute for Public Health, University of Calgary, Calgary, Canada; ${ }^{4}$ Department of Pediatrics, Cumming School of Medicine, University of Calgary, Calgary, Canada; ${ }^{5}$ Department of Physical Therapy, Faculty of Medicine, University of British Columbia, Vancouver, Canada; ${ }^{6}$ Department of Community Health Sciences, Cumming School of Medicine, University of Calgary, Calgary, Canada

10.1136/bjsports-2021-IOC.118

Background Few investigations utilize evidence-informed preparticipation evaluation, inclusive injury definitions, and prospective surveillance to identify risk factors for dance-related injury.

Objective To identify baseline injury risk factors that may be associated with dance-related musculoskeletal (MSK) complaints in pre-professional dancers.

Design Prospective cohort study.

Setting Pre-professional ballet school; university dance program.

Participants Dancers registered in full-time ballet $[n=85,77$ females, median (range) age 15 years (11-19)] and contemporary $[n=60,58$ females, 19 years $(17-30)]$ training.

Assessment of Risk Factors Pre-participation evaluation comprised of one-year injury history (yes/no), previous training (years), Athletic Coping Skills Inventory-28 (ACSI;score), body mass index $\left(\mathrm{BMI} ; \mathrm{kg} / \mathrm{m}^{2}\right)$, total bone mineral density $\left(\mathrm{g} / \mathrm{cm}^{2}\right)$, ankle plantar/dorsiflexion (degrees), active standing turnout (degrees), three lumbopelvic control tasks (high/low risk), unipedal dynamic balance (seconds), Y-Balance Test ( $\mathrm{cm})$. Weekly dance hours were self-reported throughout one academic year. Main Outcome Measurements Self-reported MSK complaints (any physical complaint leading to difficulties participating in dance, regardless of consequences) were captured weekly by online modified Oslo Sports Trauma Research Centre's Questionnaire on Health Problems during academic year. MSK complaints were recorded (yes/no) for each participant for each week.

Results Response rate was 99\%, with 81\% (117/145) of dancers reporting at least one MSK complaint. Of the 1521 complaints (19\% first-time, $81 \%$ recurrent), ankle (22\%), knee $(21 \%)$, and foot $(12 \%)$ accounted for majority. Potential factors were identified through systematic review and a generalized linear mixed model was used to analyze the binary outcome measure. Injury history [Odds Ratio (OR) 7.37; 95\% CI $(3.41,15.91)]$ and previous week's dance hours [OR 1.02; $95 \%$ CI $(1.01,1.03)]$ were significantly associated with MSK complaint.

Conclusions Prevalence of MSK complaints amongst pre-professional ballet and contemporary dancers is high and significantly associated with injury history and training volume.
Future studies implementing injury prevention should incorporate training load monitoring to address the dynamic, recursive nature of dance injury etiology.

\section{ABSTRACT WITHDRAWN}

\section{MUSCULOSKELETAL INJURIES AND DANCE EXPOSURE ACROSS THREE YEARS IN ELITE ADOLESCENT BALLET DANCERS: IS THERE A PATTERN?}

'Valeriya Volkova, 1,2,3 Sarah J Kenny. 'Sport Injury Prevention Research Centre, Faculty of Kinesiology, University of Calgary, Calgary, Canada; ${ }^{2}$ Alberta Children's Hospital Research Institute, University of Calgary, Calgary, Canada; ${ }^{3} \mathrm{O}^{\prime}$ Brien Institute for Public Health, University of Calgary, Calgary, Canada

\subsection{6/bjsports-2021-IOC.119}

Background Dance is a popular activity among youth at all levels and involves large volumes of training. Reported injury estimates are high in this population, warranting investigation of training volume and injury patterns throughout multiple training years.

Objective To describe musculoskeletal (MSK) injuries and dance exposure in elite adolescent ballet dancers across three training seasons.

Design Prospective cohort.

Setting Vocational ballet school.

Patients (or Participants) Dancers registered in full-time elitelevel ballet training $[\mathrm{n}=172 ; 152$ female, median (range) age 15 years $(10-21)]$.

Interventions (or Assessment of Risk Factors) Volume of dance training (hours) was self-reported weekly via online questionnaire.

Main Outcome Measurements Dance-related MSK injuries were registered by self-report (weekly, online modified Oslo Sports Trauma Research Centre Questionnaire on Health Problems). Three injury definitions were utilized: physical complaints (any complaint leading to difficulty participating), medical attention (any complaint resulting in care from a clinician), time-loss (unable to complete $\geq 1$ class/rehearsal/performance for $\geq 1$ day(s)). Means (SD) for weekly dance hours and weekly injury prevalence (proportion of dancers injured/week) were estimated.

Results Questionnaire response rates ranged from 89\% (year 3) to $99 \%$ (year 1). Across three training years, dance volume ranged from 19.26 hours/week (SD:4.59) (year 3) to 22.17 (SD:5.28) (year 2), mean weekly injury prevalence ranged from $21 \%$ (year 3) to $31 \%$ (year 1) (physical complaints); $15 \%$ (years $2 \& 3$ ) to $24 \%$ (year 1) (medical attention), and $5 \%$ (years 1\&3) to $9 \%$ (year 2) (time-loss). Weekly selfreported injuries mirrored the fluctuations in weekly dance exposure across the three years.

Conclusions Elite adolescent dancers report long hours of weekly training with variations across a training season. When defined as a physical complaint, dance-related MSK injury estimates are high in this population. As dance exposure hours increase, so do self-reported injury estimates. Further research is needed to explore associations between intensity of dance exposure (i.e., training load) and injuries to inform development of effective injury prevention strategies for this high-risk group of artistic athletes. 